\title{
New Opportunities in Advanced Breast Cancer
}

\author{
Thomas Decker ${ }^{\mathrm{a}}$ Marcus Schmidt ${ }^{\mathrm{b}}$

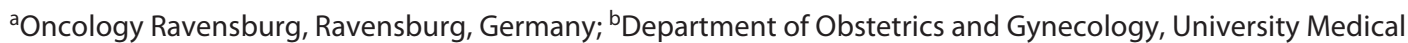 \\ Center Mainz, Mainz, Germany
}

\section{Keywords}

Advanced breast cancer - Antibody-drug-conjugates . Chemotherapy · Endocrine therapy · Patient-reported outcomes · Positron emission tomography

Breast cancer is the most common cancer type in women. Despite considerable advances in diagnosis and treatment, it is also the leading cause of cancer deaths in women. Metastatic breast cancer is an incurable but treatable disease. The main therapeutic goals are, on the one hand, the control of the disease with delay of progression and, on the other hand, control of symptoms and the preservation of the quality of life (QoL). It is therefore consensus in national and international guidelines and recommendations that endocrine-based therapy should be the first-line therapy for patients with metastatic hormone receptor-positive and human epidermal growth factor receptor 2 (HER2)-negative breast cancer, unless the patient's clinical symptoms or wishes suggest other treatments. Endocrine therapies such as aromatase inhibitors or fulvestrant are combined with cyclin-dependent kinase 4/6 inhibitors. These endocrine-based therapies have already entered daily clinical practice. In this issue, focusing on advanced breast cancer, we focus on diagnostic and therapeutic modalities that have not yet arrived in daily clinical practice to this extent.

In patients with HER2-positive or triple-negative breast cancer, novel antibody-drug-conjugates (ADCs) are receiving increased attention. These compounds consist of a monoclonal antibody with a well-defined target (e.g., HER2 or TROP-2). A cytotoxic compound is bound to this antibody with a linker. After the ADC has reached its target cell, the cytotoxic compound is released and destroys the target cell. This new therapeutic principle is very promising and will improve our therapeutic armamentarium through increased efficacy and an improved toxicity profile. A recent review paper in this issue (Marmé, Antibody-drug-conjugates for breast cancer) describes in more detail the mechanism and potential pitfalls of this new class of therapies. It focusses on the clinical experience with ADCs approved in breast cancer as well as promising candidates in late-stage clinical development. Furthermore, the mode of action, biology, and composition of ADCs and how each of these crucial components influences their properties and efficacy are discussed.

Although ADCs show great promise in advanced breast cancer, conventional chemotherapies remain a mainstay of treatment. Krajnak and co-workers (Metronomic chemotherapy for metastatic breast cancer) describe in this issue the principle of low-dose metronomic chemotherapy in advanced breast cancer. In this approach, daily metronomic dosing results in novel antiangiogenic and immunomodulatory effects that may improve efficacy in heavily treated patients with a good toxicity profile. Due to the multimodal mechanisms of action, the combination with targeted and immunological therapies may represent a new promising approach for the treatment of metastatic breast cancer. The balance between efficacy and toxicity is critical in the treatment of patients with advanced cancer.

Equally important is the search for new ways to better target therapies with little burden on the patient. In this context, liquid biopsy, which measures circulating tumor cells and/or circulating tumor DNA, offers new opportunities not only to assess tumor response but also to mon- 
itor changes in tumor cell biology (e.g., changes in receptor status or acquired mutations) in real time. BanysPaluchowski et al. (Liquid biopsy in metastatic breast cancer: current role of circulating tumor cells and circulating tumor DNA) address in this issue this increasingly important topic, which could lead to improved individualization of therapeutic strategies. However, liquid biopsy is not the only method with the potential to detect a tumor response earlier compared to conventional diagnostic measures.

Positron emission tomography (PET) is a method that not only offers the possibility to measure the diameter of metastases but also to assess metabolic activity. In this issue, Pabst et al. (The future Role of PET Imaging in Metastatic Breast Cancer) address new tracers and applications of PET/CT imaging, as well as therapeutic approaches in patients with advanced breast cancer. They also provide an outlook on the application of artificial intelligence, immunoPET, and liquid biopsy.

However, in a palliative setting of a treatable but incurable disease such as advanced breast cancer, QoL is of critical importance to patients. In this context, Hillebrand and colleagues (The Significance of Patient-Reported Outcomes for Metastatic Breast Cancer Patients) focus on the increasingly important topic of patient-reported outcomes (PROs). PROs can be used for shared treatment decisions, symptom management and control, and improvement of QoL. Regulatory agencies are increasingly considering PROs when approving new drugs. Therefore, the inclusion of PROs in metastatic breast cancer is of significant value.
On behalf of the editorial team, we hope that this issue, which focuses on diagnostic and therapeutic modalities that are not yet mainstream, will give readers a glimpse into the fascinating world of breast cancer care and encourage them to occasionally stray from well-trodden paths.

\section{Conflict of Interest Statement}

Thomas Decker received honoraria from Iomedico and advisory board honoraria from Novartis and Roche. Marcus Schmidt reports personal fees from AstraZeneca, BioNTech, Eisai, Lilly, MSD, Novartis, Pantarhei Bioscience, Pfizer, Roche, and SeaGen, and institutional research funding from AstraZeneca, BioNTech, Eisai, Genentech, German Breast Group, Novartis, Palleos, Pantarhei Bioscience, Pierre-Fabre, Roche, and SeaGen. In addition, he is named as an inventor on patent EP $2390370 \mathrm{~B} 1$ and patent EP 2951317 B1

\section{Funding Sources}

The authors did not receive grants from any funding agency in the public, commercial, or not-for-profit sectors.

\section{Author Contributions}

Thomas Decker and Marcus Schmidt drafted the manuscript. 